\title{
Perfil de la investigación sobre inteligencia de negocios en América Latina
}

\section{Profile of Research on Business Intelligence Solutions in Latin America}

\author{
A. Bustamante', E. Galvis², L.C. Gómez ${ }^{3}$ \\ ${ }^{1}$ Grupo STI, Escuela de Ingeniería de Sistemas, Universidad Industrial de Santander, Colombia. Email: abustamante@unimagdalena.edu.co \\ ${ }^{2}$ Grupo STI, Ingeniería de Sistemas, Universidad del Magdalena, Colombia. Email: egarciap@unimagdalena.edu.co \\ ${ }^{3}$ Grupo STI, Escuela de Ingeniería de Sistemas, Universidad Industrial de Santander, Colombia. Email: lcgomezf@uis.edu.co
}

RECIBIDO: Octubre 8, 2014. ACEPTADO: Diciembre 15, 2015. VERSIÓN FINAL: Abril 22, 2016

DOI: http://dx.doi.org/10.18273/revuin.v15n1-2016004 @) ब

\begin{abstract}
RESUMEN
Este estudio presenta un análisis detallado de la investigación en el área de la Inteligencia de Negocios en América Latina, durante el periodo 2000 - 2012. Los datos de este estudio fueron extraídos de la base de datos de resumes y citaciones bibliográficas SCOPUS y analizados siguiendo la metodología propuesta por Alan Porter. El software utilizado para soportar las técnicas de análisis cienciométrico fue The Vantage Point. Los resultados muestran un crecimiento en la producción de la literatura científica sobre Inteligencia de Negocios, que ha aumentado de 22 artículos en el 2000 a 261 en el 2012. Adicionalmente, se evidencia que los temas más investigados están relacionados con la minería de datos y que las instituciones de Brasil producen la mayor cantidad de la literatura en la región, pues el $56 \%$ de las publicaciones provienen de ese país.
\end{abstract}

Palabras Clave: América Latina, Cienciométria, Inteligencia de negocios.

\section{ABSTRACT}

This study presents a detailed analysis of research developed on Business Intelligence in Latin America, between 2000 and 2012. The data of this study was taken from the largest abstract and citation database of peer-reviewed literature, SCOPUS. It was analysed using the methodology proposed by Alan Porter. The software used to support the scientometric analysis techniques was The Vantage Point. The results show an increasing growth in literature on Business Intelligence, which has increased from 22 items in 2000 to 261 in 2012. Additionally, it is evident that the most of the researched topics are related with data mining and that Brazilian Institutions produce most of the literature in the region: approximately $56 \%$ of the publications come from this country.

KEYWORDS: Business Intelligence, Latin America, Scientometric.

\section{INTRODUCCIÓN}

La inteligencia de negocios es un concepto que emergió en la década de los 90's como un tipo de sistema de información que buscaba proporcionar la arquitectura tecnológica necesaria para aprovechar los datos operacionales generados por las organizaciones [1]. En otras palabras, esta tecnología buscaba tratar los datos para transformarlos en información, luego en conocimiento y así utilizarlos en el proceso de toma de decisiones, de forma que se lograra obtener y mantener ventaja competitiva [2]. En la última década este tipo de sistemas de información ha ganado madurez, pasando a ser la evolución de los sistemas de soporte a la toma de decisiones tradicionales [3], y una de las tecnologías de información de mayor crecimiento en el área de los sistemas de soporte a la toma de decisión, según lo revelan informes de firmas internacionales como [4] y [5].

Este artículo puede compartirse bajo la licencia CC BY-ND 4.0 (https://creativecommons.org/licenses/by-nd/4.0/) y debe referenciarse usando el siguiente formato: A. Bustamante et al., "Perfil de la investigación sobre inteligencia de negocios en América Latina," UIS Ingenierias, vol. I5, no. I, pp. 4I-5I, May. 2016. 
La madurez y evolución logradas han sido el resultado de todas las investigaciones y desarrollos tecnológicos en temas como bodegas de datos y minería de datos. Sin embargo, aún existen diversos desafíos que requieren mayor investigación. Uno de estos es incorporar la información no estructurada en el proceso de toma de decisiones [6].

Dicho lo anterior, el propósito de este artículo es presentar un perfil de la investigación en el área de Inteligencia de Negocios en América Latina, con el objetivo de determinar la capacidad y fortaleza investigativa de la región en esta área. Este tipo de estudio cienciométrico se ha hecho en otras áreas de la computación como la Computación en la Nube [7], el Comercio Electrónico [8] y los Sistemas de Información Geográfica [9].

Particularmente, este perfil de la investigación da respuesta a preguntas como: ¿Qué países e instituciones presentan mayor número de publicaciones? ¿Quiénes son los principales investigadores? ¿Cuáles son los temas más investigados y la relación que existe entre ellos? Con este acercamiento, los profesionales e investigadores en el área disponen de datos que les permiten identificar nuevos temas de investigación o nuevos contactos para desarrollar iniciativas de investigación.

Este estudio de investigación cienciométrica es posible gracias a hechos como la consolidación de las bases de datos de resúmenes e indización científica, la variedad de metadatos que estas bases de datos proveen, la gran cantidad de información generada procedente de investigación; y la necesidad de monitorear la evolución, la consolidación, el esparcimiento, la mutación, o la desaparición de la tecnología.

La ventaja de usar las herramientas y técnicas para análisis cienciométrico radica en que se puede tener una visión general del estado de desarrollo de un área, campo o tecnología, sin necesidad de revisar en detalle toda la literatura disponible. Particularmente, se busca identificar el nuevo conocimiento generado en las comunidades científicas para revelar tendencias, de modo que la información resultante pueda ser usada para tomar decisiones respecto a qué se debe investigar, con quién se deben establecer relaciones para investigar en un área específica y dónde se debe buscar la información pertinente para llevar a cabo las investigaciones.

\section{Metodología}

La metodología utilizada para llevar a cabo el análisis fue la propuesta por [10]. Esta metodología se compone de nueve pasos agrupados en tres fases: inteligencia, análisis y diseño, y selección; tal como se indica en la Tabla I.

En la fase de inteligencia se precisó el concepto Inteligencia de Negocios, como tema a analizar; el periodo comprendido entre 2000 y 2012, como frontera temporal; y los países de América Latina, como frontera geográfica. También se definió como fuente de información la base de datos de citaciones y resúmenes SCOPUS, en dónde se realizaron varias iteraciones con términos y operadores de búsqueda que permitieron definir la ecuación de búsqueda que se presenta en la Figura 1.

La fase de análisis y diseño inició con la limpieza y unificación de los datos. Se unificaron y corrigieron inconsistencias en los nombres de autores, las instituciones y en las palabras claves. Luego, se construyeron tablas de frecuencia por autor, país e institución y tablas de contingencia para comparar las variables mencionadas. Posteriormente, se crearon mapas de conexión entre las palabras claves y los conceptos presentes en los títulos y resúmenes.

Finalmente, durante la fase de selección se construyeron gráficos para apreciar fácilmente la variabilidad de las publicaciones. Para esto, se hicieron cambios en el formato de los resultados arrojados por el software utilizado. También se realizaron inferencias con base en los resultados obtenidos y por último, se construyó este artículo como reporte final del resultado de la investigación.

\section{Resultados}

El primer hallazgo fue la identificación de una tendencia de crecimiento en el número de publicaciones. Como se muestra en la Figura 2, se pasó de 22 publicaciones en el año 2000 a 261 en el 2012. Esto equivale a un crecimiento aproximado del 1086\%. Sin embargo, como se muestra en la Figura 3, esta tendencia de crecimiento ha estado jalonada principalmente por la producción de Brasil, México y Chile. En los demás países dentro del top 10 de producción en la región, el crecimiento no es tan elevado. 
TABLA I

Resumen De Recolección De Información

\begin{tabular}{|c|c|c|}
\hline Fases & Pasos & Objetivo \\
\hline \multirow{3}{*}{ Inteligencia } & Identificación del tema & $\begin{array}{l}\text { Definir el área, campo o concepto a analizar, la frontera temporal y geográfica, y las preguntas } \\
\text { guías del proceso. }\end{array}$ \\
\hline & $\begin{array}{l}\text { Selección de la fuentes de } \\
\text { Información }\end{array}$ & $\begin{array}{l}\text { Hacer explicitas las fuentes de información que se utilizarán para recuperar los metadatos y } \\
\text { datos a analizar. }\end{array}$ \\
\hline & $\begin{array}{l}\text { Refinamiento de la } \\
\text { búsqueda y recuperación } \\
\text { de la información }\end{array}$ & $\begin{array}{l}\text { Establecer la(s) ecuación(es) a utilizar para recuperar la información. Con base en la revisión } \\
\text { de los resultados se entra en un proceso iterativo de calibración de la ecuación, con base en } \\
\text { los resultados. }\end{array}$ \\
\hline \multirow{3}{*}{$\begin{array}{l}\text { Análisis y } \\
\text { diseño }\end{array}$} & Limpieza de los datos & $\begin{array}{l}\text { Eliminar la redundancia y las variaciones innecesarias en los datos. Para esto se depuran los } \\
\text { metadatos y datos, dejando estos en un formato apropiado para ser utilizado por la } \\
\text { herramienta de análisis. }\end{array}$ \\
\hline & Análisis básico & $\begin{array}{l}\text { Empezar el análisis de los datos a través de un análisis exploratorio, la limpieza de datos } \\
\text { (adicional al paso dos y como resultado del análisis exploratorio), el análisis de primer orden } \\
\text { (producir listas) y el análisis de segundo orden (producir de matrices). }\end{array}$ \\
\hline & Análisis avanzado & Analizar el grado de conexión entre los diferentes elementos. \\
\hline \multirow{3}{*}{ Selección } & Representación & Escoger la forma de presentar los datos dependiendo de la población objetivo del estudio. \\
\hline & Interpretación & Hacer inferencias con base en los resultados obtenidos. \\
\hline & Utilización & $\begin{array}{l}\text { Usar los resultados y plasmar las inferencias en informes, documentos técnicos, etc. Hecho } \\
\text { esto, queda que se utilicen por parte de los usuarios como soporte o insumo de sus decisiones. }\end{array}$ \\
\hline
\end{tabular}

Fuente: Elaboración propia con base en [10].

(TITLE-ABS-KEY("Business Intelligence" OR "Data Warehouse" OR "DW" OR "On-Line Analytical Processing" OR "OLAP" OR "Multidimensional Database" OR "Data Mart" OR "Decision support database" OR "Decision support System" OR "Multidimensional Modeling" OR "Multidimensional Design" OR "Multidimensional Data Modeling" OR "multidimensional data Model" OR "multidimensional Model" OR "Data cube" OR "Data mining" OR "scorecard" OR "dashboard" OR "KPI" OR "Key Performance Indicator" OR "ETL" OR "Extraction Transformation and Load")) AND (AFFILCOUNTRY("Argentina" OR "Bolivia" OR "Brazil" OR "Chile" OR "Colombia" OR "Costa Rica" OR "Cuba" OR "Ecuador" OR "El Salvador" OR "Guatemala" OR "Haiti" OR "Honduras" OR "Mexico" OR "Nicaragua" OR "Panama" OR "Paraguay" OR "Peru" OR "Dominican Republic" OR "Uruguay" OR "Venezuela" OR "Puerto Rico")) and ( (PUBYEAR > 1999) and ( PUBYEAR < 2013))

Figura I. Ecuación de búsqueda. Fuente. Elaboración propia 


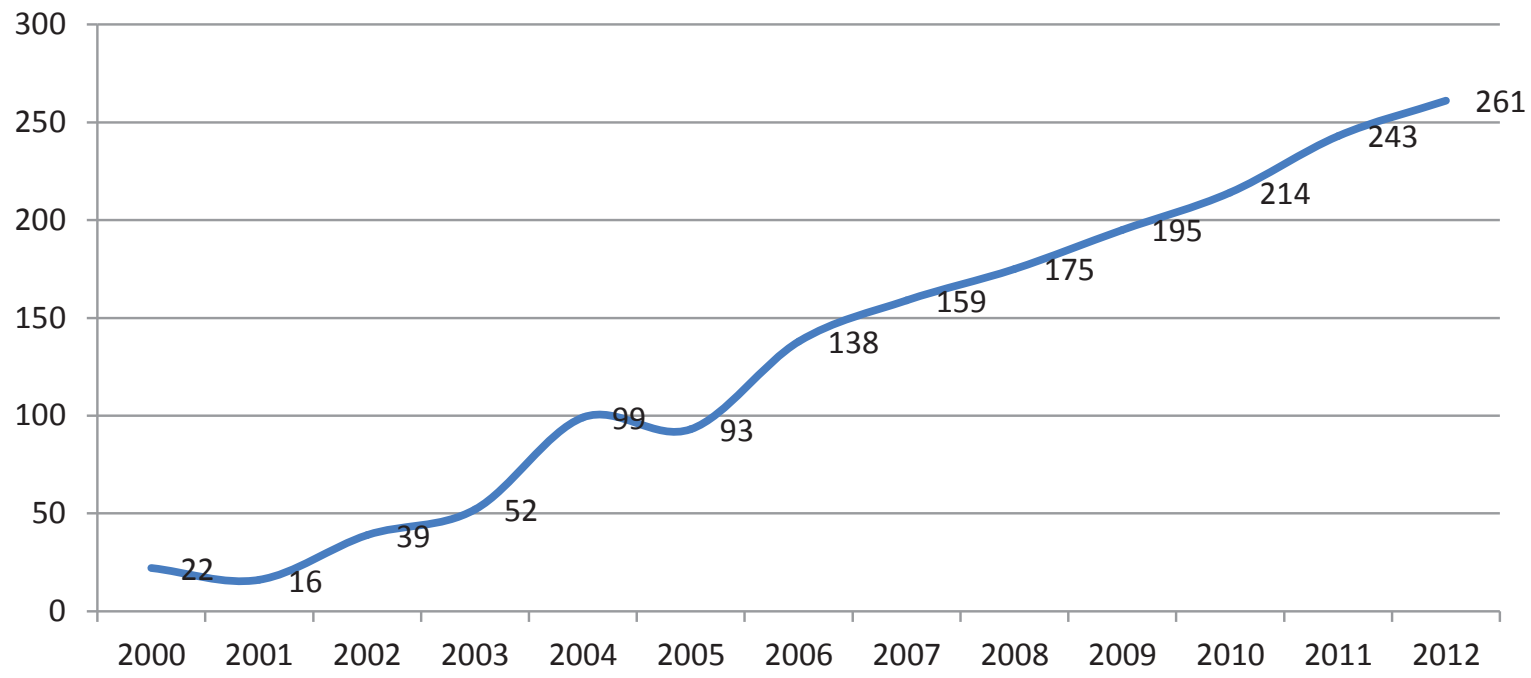

Figura 2. Comportamiento anual de la cantidad de publicaciones durante el periodo 2000 - 20I2. Fuente. Elaboración propia.

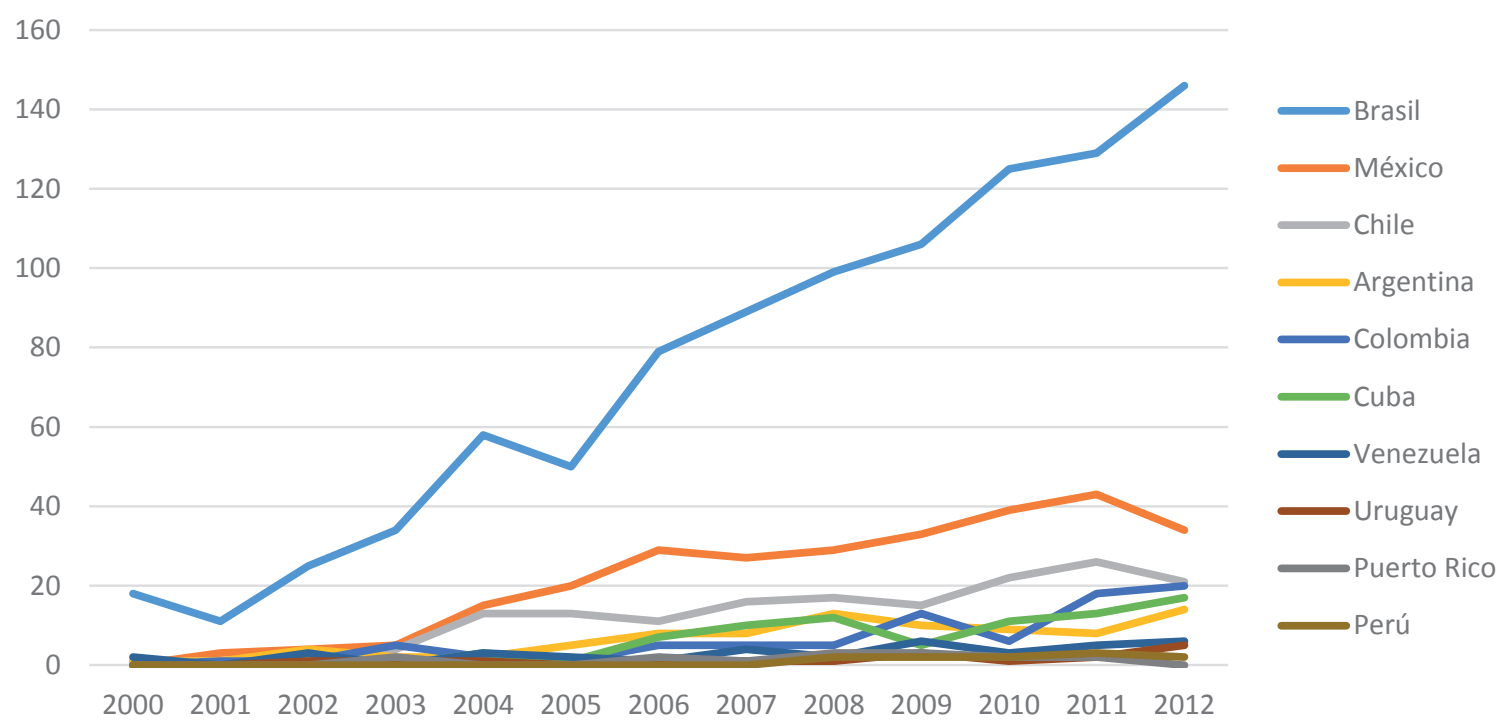

Figura 3. Comportamiento anual por país de la cantidad de publicaciones durante el periodo 2000 - 2012.

Fuente. Elaboración propia.

Así mismo, en la Tabla II se puede apreciar que la producción de Brasil es mayor que la suma de la producción de todos los demás países latinoamericanos, siendo equivalente al $56 \%$ de la totalidad. México y Chile realizan un aporte significativo con el $16 \%$ y $9 \%$ respectivamente. Argentina, Colombia y Cuba rondan el $4 \%$ cada uno; mientras que los países restantes: Venezuela, Uruguay, Puerto Rico y Perú suman entre todos, aproximadamente el $4 \%$ de la producción.
Como lo muestran las Tablas III y IV, de los países no Latinoamericanos, España y Estados Unidos han realizado el mayor aporte, superando entre los dos el 50\% de la producción total hecha en colaboración. Llama la atención que si se incluyera la producción de España y Estados Unidos en la Tabla II, tomando como referencia únicamente las publicaciones en las que han colaborado autores de estos países, estas ocuparían el cuarto y quinto 
puesto con el $9 \%$ y el $8 \%$ respectivamente, superando a países de la región como Argentina, Colombia y Cuba, que representan el 4\% individualmente.

Igualmente, en la Tabla IV se observa que aproximadamente el 30\%, de la producción de los países del escalafón es realizada en cooperación internacional, siendo Brasil, México y Chile quienes realizan mayor trabajo cooperativo, con el $25 \%, \quad 35 \%$ y $57 \%$ respectivamente.

También se encontró que los autores con mayor producción están asociados a instituciones de Brasil. En la Tabla $\mathrm{V}$ se presentan los 10 autores con mayor producción, con un predominio claro de los afiliados a universidades de Brasil.

TABLA II

Distribución De Publicaciones Entre Países latinoamericanos Durante El Periodo 2002-20I2

\begin{tabular}{|c|c|c|c|c|c|c|c|c|c|c|c|c|c|c|}
\hline País/Año & 2000 & 2001 & 2002 & 2003 & 2004 & 2005 & 2006 & 2007 & 2008 & 2009 & 2010 & 2011 & 2012 & Total \\
\hline Brasil & 18 & II & 25 & 34 & 58 & 50 & 79 & 89 & 99 & 106 & 125 & 129 & 146 & 969 \\
\hline México & - & 3 & 4 & 5 & 15 & 20 & 29 & 27 & 29 & 33 & 39 & 43 & 34 & 281 \\
\hline Chile & $\mathrm{I}$ & 1 & 1 & 4 & 13 & 13 & 11 & 16 & 17 & 15 & 22 & 26 & 21 & 161 \\
\hline Argentina & I & I & 4 & 2 & 2 & 5 & 8 & 8 & 13 & 10 & 9 & 8 & 14 & 85 \\
\hline Colombia & - & I & $\mathrm{I}$ & 5 & 2 & 1 & 5 & 5 & 5 & 13 & 6 & 18 & 20 & 82 \\
\hline Cuba & - & - & 1 & - & 3 & 1 & 7 & 10 & 12 & 5 & II & 13 & 17 & 80 \\
\hline Venezuela & 2 & - & 3 & - & 3 & 2 & $\mathrm{I}$ & 4 & 2 & 6 & 3 & 5 & 6 & 37 \\
\hline Uruguay & - & - & 1 & 1 & 1 & - & $\mathrm{I}$ & $\mathrm{I}$ & I & 3 & $\mathrm{I}$ & 2 & 5 & 17 \\
\hline Puerto Rico & - & - & - & 2 & - & - & 2 & I & 3 & 3 & 2 & 2 & - & 15 \\
\hline Perú & - & - & - & - & - & - & - & - & 2 & 2 & 2 & 3 & 2 & II \\
\hline Total & 22 & 17 & 40 & 53 & 97 & 92 & 143 & 161 & 183 & 196 & 220 & 249 & 265 & 1738 \\
\hline
\end{tabular}

Fuente. Elaboración propia

TABLA III

Distribución De Publicaciones Entre Países No latinoamericanos Durante El Periodo 2002-20 I2

\begin{tabular}{|c|c|c|c|c|c|c|c|c|c|c|c|c|c|c|}
\hline País/Año & 2000 & 2001 & 2002 & 2003 & 2004 & 2005 & 2006 & 2007 & 2008 & 2009 & 2010 & 2011 & 2012 & Total \\
\hline España & - & - & 2 & 3 & 9 & 9 & 15 & 17 & 19 & 20 & 21 & 22 & 26 & 163 \\
\hline Estados Unidos & - & I & 5 & 6 & 13 & 8 & 17 & 13 & II & II & 23 & 20 & 23 & $|5|$ \\
\hline Reino Unido (UK) & I & - & $\mathrm{I}$ & 2 & 8 & 2 & 2 & 8 & 3 & 8 & 5 & 7 & 7 & 54 \\
\hline Francia & I & I & I & 2 & 5 & 3 & 5 & 5 & 7 & 5 & 8 & 4 & 4 & 51 \\
\hline Alemania & - & - & 2 & - & 2 & 3 & $\mathrm{I}$ & 3 & 5 & 5 & 6 & 7 & 9 & 43 \\
\hline Bélgica & - & - & - & - & 4 & 2 & 4 & 2 & 7 & 5 & I & 6 & 4 & 35 \\
\hline Canadá & I & - & - & $\mathrm{I}$ & 2 & 2 & 5 & 2 & 2 & 4 & $\mathrm{I}$ & 4 & 5 & 29 \\
\hline Italia & - & - & $\mathrm{I}$ & - & - & - & - & 4 & - & 3 & 4 & 6 & 6 & 24 \\
\hline Australia & - & - & I & - & I & - & I & I & 4 & - & I & 5 & 2 & 16 \\
\hline Portugal & - & - & - & - & I & $\mathrm{I}$ & - & I & 2 & I & - & 3 & 6 & 15 \\
\hline Total & 3 & 2 & 13 & 14 & 45 & 30 & 50 & 56 & 60 & 62 & 70 & 84 & 92 & 581 \\
\hline
\end{tabular}

Fuente. Elaboración propia

TABLA IV

Cooperación Entre Países latinoamericanos Y No latinoamericanos Durante El Periodo 2002-20I2

\begin{tabular}{|c|c|c|c|c|c|c|c|c|c|c|c|}
\hline País/País & España & USA & UK & Francia & Alemania & Bélgica & Canadá & Italia & Australia & Portugal & Total \\
\hline Brasil & 23 & 68 & 36 & 31 & 21 & 9 & 16 & II & 4 & 15 & 234 \\
\hline México & 32 & 37 & 6 & 12 & 3 & - & 3 & 4 & $\mathrm{I}$ & - & 98 \\
\hline Chile & 37 & 12 & 7 & 5 & II & 2 & 6 & 3 & 8 & 1 & 92 \\
\hline Argentina & 11 & $\mathrm{II}$ & 2 & 2 & 4 & 12 & 4 & 5 & 2 & - & 53 \\
\hline Cuba & 43 & $\mathrm{I}$ & 2 & 1 & 2 & 1 & - & 2 & - & - & 52 \\
\hline Colombia & 10 & 10 & - & 1 & 1 & 2 & - & - & 3 & - & 27 \\
\hline Venezuela & 10 & 8 & 2 & 2 & 3 & - & - & I & 2 & - & 28 \\
\hline Uruguay & 5 & $\mathrm{I}$ & $\mathrm{I}$ & 2 & - & 2 & I & - & - & - & 12 \\
\hline $\begin{array}{l}\text { Puerto } \\
\text { Rico } \\
\end{array}$ & - & 5 & - & - & - & - & I & - & - & - & 6 \\
\hline Perú & 5 & 4 & - & - & - & - & - & - & - & - & 9 \\
\hline Total & 176 & 157 & 56 & 56 & 45 & 28 & 31 & 26 & 20 & 16 & 611 \\
\hline
\end{tabular}

Fuente. Elaboración propia 


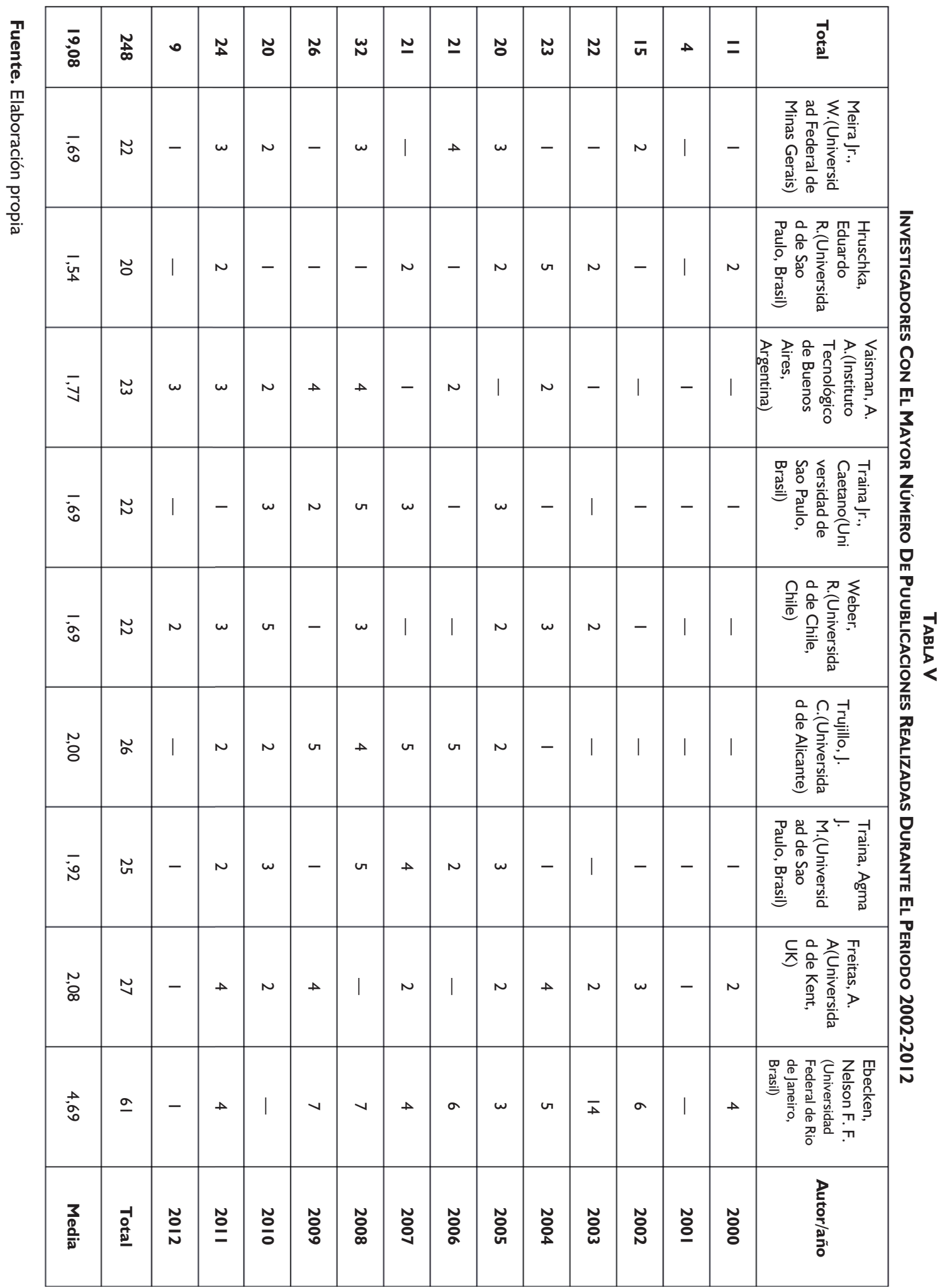


TABLA VI

Producción De UniVersidades Latinoamericanas

\begin{tabular}{|c|c|c|c|c|c|c|c|c|c|c|c|c|c|c|}
\hline Universidad/Año & 2000 & 2001 & 2002 & 2003 & 2004 & 2005 & 2006 & 2007 & 2008 & 2009 & 2010 & 2011 & 2012 & Total \\
\hline $\begin{array}{lll}\text { Universidad de } & \text { Sao } \\
\text { Paulo, Brasil } & & \\
\end{array}$ & 2 & 2 & 2 & 3 & 9 & 9 & 9 & 19 & 21 & 21 & 25 & 27 & 24 & 173 \\
\hline $\begin{array}{l}\text { Universidad Federal de } \\
\text { Rio de Janeiro, Brasil }\end{array}$ & 6 & 2 & 7 & 17 & 10 & II & 11 & 14 & 15 & 19 & 3 & 13 & 13 & $14 \mid$ \\
\hline $\begin{array}{l}\text { Universidad de Chile, } \\
\text { Chile }\end{array}$ & 1 & 1 & 一 & 3 & 6 & 9 & 4 & 5 & 6 & 6 & 12 & 10 & 9 & 72 \\
\hline UNICAMP, Brasil & 2 & 3 & I & 3 & 2 & 3 & 4 & 7 & 7 & 3 & $\mathrm{II}$ & 8 & 10 & 64 \\
\hline $\begin{array}{l}\text { Universidad Federal de } \\
\text { Pernambuco, Brasil }\end{array}$ & - & - & 1 & 1 & 3 & - & 5 & 3 & 6 & 8 & 9 & 8 & 9 & 53 \\
\hline $\begin{array}{l}\text { Universidad Federal de } \\
\text { Minas Gerais, Brasil }\end{array}$ & - & - & 1 & 2 & 3 & 4 & 5 & - & 5 & 7 & 6 & 5 & 6 & 44 \\
\hline $\begin{array}{l}\text { Universidad Federal de } \\
\text { San Carlos, Brasil }\end{array}$ & 1 & - & 1 & - & 4 & 1 & 1 & 4 & 4 & 5 & 8 & II & 7 & 47 \\
\hline $\begin{array}{l}\text { Universidad Autónoma } \\
\text { de México, México }\end{array}$ & - & $\mathrm{I}$ & - & I & 1 & 2 & 2 & 1 & 4 & 7 & 6 & 7 & 7 & 39 \\
\hline $\begin{array}{l}\text { Universidad Federal de } \\
\text { Rio Grande del Sur, } \\
\text { Brasil }\end{array}$ & - & - & 1 & 2 & 1 & 2 & 4 & 1 & 3 & 5 & 5 & 7 & 8 & 39 \\
\hline $\begin{array}{l}\text { Universidad de Buenos } \\
\text { Aires, Argentina }\end{array}$ & - & I & 2 & 2 & 2 & I & 4 & 2 & 6 & 5 & 3 & 2 & 4 & 34 \\
\hline Total & 12 & 10 & 16 & 34 & 41 & 42 & 49 & 56 & 77 & 86 & 88 & 98 & 97 & 706 \\
\hline
\end{tabular}

\section{Fuente. Elaboración propia}

Por otro lado, en la Tabla VI se puede ver que siete de las diez universidades con mayor cantidad de publicaciones en América Latina son de Brasil. Siendo la primera la Universidad de São Paulo, número uno de las universidades latinoamericanas según [11]. Otras universidades de la lista que también aparecen en el ranking, y en el siguiente orden son: la Universidad de Buenos Aires (UBA), la Universidad Nacional de México (UNAM), las Universidades Federales de Minas Gerais y Río de Janeiro; la Universidad Federal de Río Grande do Sul y la Universidad de Chile. Pero más allá de esta importante presencia cabe destacar que las universidades latinoamericanas son casi invisibles en dicho ranking, y que la primera, ya mencionada, aparece en el rango de las 101-150.

Los términos contenidos en la Tabla VII y la Figura 4 emergen del análisis hecho con el software Vantage Point sobre las palabras claves y los términos extraídos de los resúmenes, a través de las herramientas de procesamiento de texto. En relación a los temas, los temas que más se investigan en la región latinoamericana son: minería de datos, bodegas de datos y ontologías.

Para el caso de la minería de datos, se observa que además de ser el tema más investigado, se aborda desde distintas perspectivas, tales como 'Data Mining Technique', 'Data Mining Method' y 'Data Mining Tools'.
Un fenómeno similar ocurre con el tema bodegas de datos, que se aborda de una manera amplia dentro de la Tabla VII: bodegas de datos espaciales, diseño de bodega de datos u otro tema estrechamente relacionado como el Procesamiento Analítico en Línea (OLAP).

Llama la atención, que el término 'Inteligencia de Negocios' aparece en el lugar número doce, siendo superado por tecnologías que suelen están contenidas dentro de este término sombrilla.

Sumado a esto, en la Figura 4 se ilustra la conexión existente entre los diferentes temas de interés relacionados con la Inteligencia de Negocios, tales como, 'Bodegas de datos', 'Procesos de ETL', 'Sistemas de soporte a la toma de decisiones', 'Bases de datos', 'Minería de textos', entre otros. En este sentido, los términos que presentan conexión directa con la Inteligencia de Negocios son 'Planeación estratégica', 'Inteligencia competitiva', 'Procesos de ETL' y 'Bodegas de datos' en sus diferentes variables.

En último lugar, se observó que hay términos como 'Ingeniería del software' y 'Gestión del conocimiento software', que aparecen dentro de la figura, pero que no se conectan directamente con los términos 'Inteligencia de negocios', 'Bodega de datos' y 'Minería de datos' que aparecen dentro de la misma figura. 
TABLA VII

Coocurrencia De Temas Investigados

\begin{tabular}{|c|c|c|c|c|c|c|c|c|c|c|c|c|c|c|c|c|c|c|c|c|c|c|c|c|}
\hline & 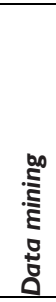 & 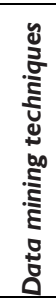 & 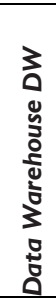 & 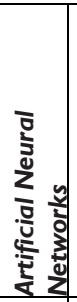 & 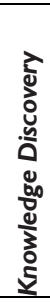 & 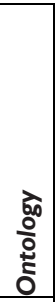 & 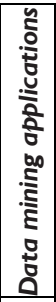 & 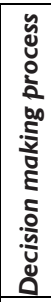 & 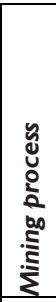 & 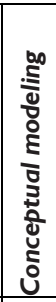 & 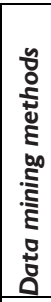 & 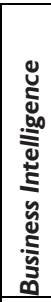 & 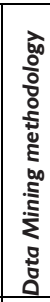 & 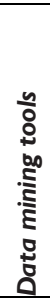 & 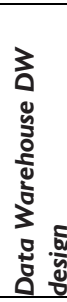 & 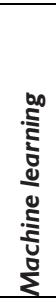 & 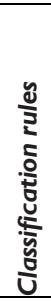 & 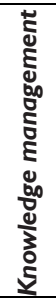 & 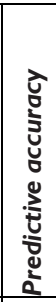 & 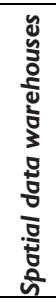 & 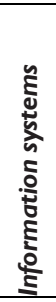 & 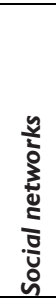 & $\frac{2}{5}$ & 퓸 \\
\hline Data mining & 327 & 30 & 5 & \begin{tabular}{l|}
15 \\
\end{tabular} & 24 & 7 & $\mathrm{I}$ & 3 & 2 & 1 & 2 & 4 & 6 & 4 & - & 13 & 8 & 4 & 5 & - & 3 & 4 & $\mathrm{I}$ & 469 \\
\hline $\begin{array}{l}\text { Data mining } \\
\text { techniques }\end{array}$ & 30 & 159 & 2 & 10 & 14 & 8 & 3 & - & 3 & - & 2 & - & 6 & 2 & - & 1 & 3 & I & - & - & I & 4 & - & 249 \\
\hline $\begin{array}{l}\text { Data } \\
\text { Warehouse } \\
\text { DW }\end{array}$ & 5 & 2 & 118 & I & 5 & 4 & - & 7 & - & 10 & I & 2 & - & - & 19 & I & - & - & - & I & 2 & - & I & 179 \\
\hline $\begin{array}{l}\text { Artificial } \\
\text { Neural } \\
\text { Networks }\end{array}$ & 15 & 9 & I & 73 & 5 & - & 4 & - & - & - & 2 & - & I & 2 & - & 3 & 3 & - & I & - & - & - & - & 119 \\
\hline $\begin{array}{l}\text { Knowledge } \\
\text { Discovery }\end{array}$ & 24 & 12 & 5 & 5 & 71 & I & I & I & - & - & 2 & I & 2 & I & - & - & I & I & 3 & - & I & - & - & 132 \\
\hline Ontology & 7 & 7 & 4 & - & $\mathrm{I}$ & 42 & $\mathrm{I}$ & - & - & 2 & - & $\mathrm{I}$ & - & $\mathrm{I}$ & - & $\mathrm{I}$ & - & $\mathrm{I}$ & - & - & - & - & - & 68 \\
\hline $\begin{array}{l}\text { Data mining } \\
\text { applications }\end{array}$ & I & I & - & 4 & I & 1 & 31 & - & - & - & I & - & - & I & - & 1 & 2 & - & - & - & - & - & - & 44 \\
\hline $\begin{array}{l}\text { Decision } \\
\text { making } \\
\text { process }\end{array}$ & 3 & - & 7 & - & I & - & - & 26 & - & 2 & - & 1 & - & - & I & - & - & I & - & 3 & - & - & - & 45 \\
\hline $\begin{array}{l}\text { Mining } \\
\text { process }\end{array}$ & 2 & 2 & - & - & - & - & - & - & 24 & - & I & - & - & - & - & - & - & - & - & - & I & - & - & 30 \\
\hline $\begin{array}{l}\text { Conceptual } \\
\text { modeling }\end{array}$ & I & - & 10 & - & - & 2 & - & 2 & - & 24 & - & - & - & - & 5 & - & - & - & - & - & 2 & - & - & 46 \\
\hline $\begin{array}{l}\text { Data mining } \\
\text { methods }\end{array}$ & 2 & I & I & 2 & 2 & & I & - & 1 & - & 24 & - & - & - & - & - & I & - & I & - & - & - & - & 36 \\
\hline $\begin{array}{l}\text { Business } \\
\text { Intelligence } \\
\end{array}$ & 4 & - & 2 & - & I & I & - & I & - & - & - & 24 & - & - & I & - & - & 4 & - & - & 2 & - & - & 40 \\
\hline $\begin{array}{l}\text { Data Mining } \\
\text { methodology }\end{array}$ & 6 & 6 & - & I & 2 & - & - & - & - & - & - & - & 23 & - & - & - & I & - & - & - & - & - & - & 39 \\
\hline $\begin{array}{l}\text { Data mining } \\
\text { tools }\end{array}$ & 4 & 2 & - & 2 & I & I & I & - & - & - & - & - & - & 22 & - & - & - & - & - & - & I & - & - & 34 \\
\hline $\begin{array}{l}\text { Data } \\
\text { Warehouse } \\
\text { DW design }\end{array}$ & - & - & 19 & - & - & - & - & I & - & 5 & - & I & - & - & 22 & - & - & - & - & - & I & - & - & 49 \\
\hline $\begin{array}{l}\text { Machine } \\
\text { learning }\end{array}$ & 13 & I & I & 3 & - & I & I & - & - & - & - & - & - & - & - & 22 & - & - & I & - & - & - & - & 43 \\
\hline $\begin{array}{l}\text { Classification } \\
\text { rules }\end{array}$ & 8 & 3 & - & 3 & I & - & 2 & - & - & - & I & - & I & - & - & - & 21 & - & 4 & - & - & - & - & 44 \\
\hline $\begin{array}{l}\text { Knowledge } \\
\text { management }\end{array}$ & 4 & 1 & - & - & I & I & - & I & - & - & - & 4 & - & - & - & - & - & 21 & - & - & - & - & - & 33 \\
\hline $\begin{array}{l}\text { Spatial data } \\
\text { warehouses } \\
\text { SDWs }\end{array}$ & - & - & I & - & - & - & - & 3 & - & - & - & - & - & - & - & - & - & - & - & 20 & I & - & 2 & 27 \\
\hline $\begin{array}{l}\text { Information } \\
\text { systems }\end{array}$ & 3 & I & 2 & - & I & - & - & - & 1 & 2 & - & 2 & - & I & I & - & - & - & - & 1 & 20 & I & - & 36 \\
\hline $\begin{array}{l}\text { Social } \\
\text { networks }\end{array}$ & 4 & 4 & - & - & - & - & - & - & - & - & - & - & - & - & - & - & - & - & - & - & I & 18 & - & 27 \\
\hline OLAP & 1 & - & $\mathrm{I}$ & - & - & - & - & - & - & - & - & - & - & - & - & - & - & - & - & 2 & - & - & 17 & 21 \\
\hline Total & : & $\underset{\sim}{\nabla}$ & $\stackrel{\Omega}{\Sigma}$ & ్ㅗ & $\stackrel{\mathbb{m}}{\underline{H}}$ & 8 & $\stackrel{0}{+}$ & $\mathscr{4}$ & $\bar{m}$ & $\stackrel{\circ}{+}$ & $\hat{m}$ & 웅 & के & ले & g & $\mathscr{q}$ & \& & $\tilde{m}$ & ผn & $\hat{N}$ & ले & 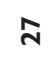 & $\bar{N}$ & \\
\hline
\end{tabular}

Fuente. Elaboración propia 


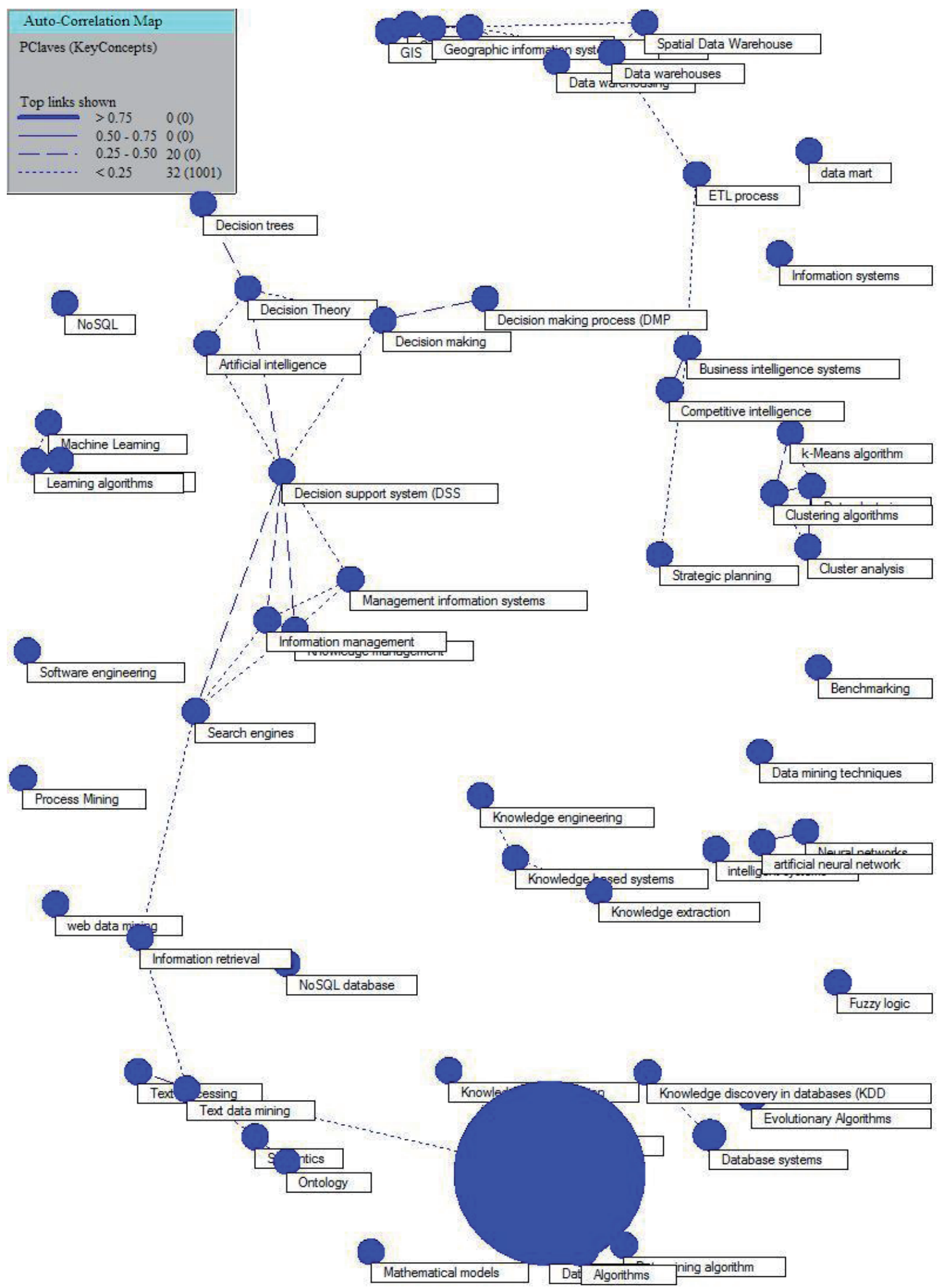

Figura 4. Mapa de distribución de temas. Fuente. Elaboración propia 


\section{Discusión}

Inicialmente se debe destacar que si bien se detectó una tendencia de crecimiento en la producción de toda la región, el incremento ha sido jalonado por un solo país: Brasil, y en los demás, parece que no existe ese mismo comportamiento. Es decir, si se extrajera a Brasil del análisis, el crecimiento no sería tan significativo, pues la tasa de crecimiento caería en un 56\% aproximadamente. El top tres de países, en términos de producción, lo conforman Brasil, México y Chile.

Las universidades mejor posicionadas en el ranking de Shanghai son las que mayor número de publicaciones tienen en el área. Es el caso de las universidades de Brasil como São Paulo; la UNAM, de México; la Universidad de Buenos Aires, en Argentina; y la Universidad de Chile.

El hecho de que aproximadamente el $30 \%$ de la publicación, de los diez países del escalafón con más publicaciones, se haya desarrollado con colaboración internacional, muestra la importancia de este tipo de interacción en la región. Esto indica que los países con volúmenes de producción más baja, pueden adoptar esta práctica, para incrementar las publicaciones en las áreas exploradas. En este mismo sentido, el trabajo conjunto entre países de la región puede ayudar a disminuir la brecha en cuanto a producción. Debido a que países con producción más baja como Perú, pueden encontrar apoyo en un país como Brasil, que ha consolidado una base de investigadores ya reconocida a nivel internacional.

De otra parte, comparando los resultados con las tendencias investigativas presentadas por el Instituto de Bodegas de Datos (TDWI - The Data Warehouse Institute), específicamente en el informe [12], que enuncia las 10 principales tendencias para el 2014, se aprecia que los temas 'Inteligencia de negocios basada en la nube', 'Inteligencia de negocios ágil', 'Inteligencia de negocios móvil' y 'Bases de datos NoSQL' son poco investigados en América Latina. Por su parte, el tema más investigado en la región, minería de datos, ocupa el quinto lugar en la lista publicada por TDWI.

De igual manera, si se considera que la Inteligencia de Negocios está en su tercera fase [13], en la que predomina la orientación a servicios, la incorporación de datos no estructurados, y el uso de tecnologías como 'Big Data', 'Hadoop', 'Ontologías', 'NoSQL', en otras, se aprecia que el trabajo realizado en América Latina no ha llegado a tratar aspectos de la tercera fase.

\section{CONCLUSIONES}

Este estudio es un primer acercamiento para determinar la capacidad de investigación de América Latina en el área de la Inteligencia de Negocios. En particular, se identificaron los países, instituciones e investigadores que presentan mayor número de publicaciones en el tema, así como, los temas más investigados y la relación que existe entre ellos.

Así mismo, se detectó que existe un incremento en las publicaciones relacionadas con el concepto de Inteligencia de Negocios en la región como conjunto y en cada país por separado. Aunque algunos países como Perú crecen a un paso lento.

En coherencia con este comportamiento, se encontró que el grupo de las 10 universidades con mayor cantidad de publicaciones en el área está conformado en un 70\% por instituciones de Brasil, lo cual evidencia el liderazgo regional de este país y sus universidades en esta área.

De igual manera, los investigadores de universidades brasileñas lideran la cantidad de aportes hechos en el área, por lo cual pueden constituirse en apoyo para universidades y centros de investigación con menor madurez.

Por otra parte, se encontró que los componentes de Inteligencia de Negocios que reciben mayor atención por parte de los investigadores son la 'minería de datos' y las 'bodegas de datos'. Igualmente, se apreció que en América Latina la investigación se distribuye entre los diferentes conceptos que este término sombrilla encierra.

También se debe destacar que el hecho de que el término 'Inteligencia de Negocios' enmarque una variedad de técnicas y métodos, dificulta el hacer un análisis más preciso con este tipo de herramientas, pues no se llega a leer el detalle del documento.

Finalmente, se espera que a partir de este estudio se desarrollen trabajos que identifiquen los mismos elementos a nivel global, con el fin de contrastar los resultados obtenidos en América Latina e identificar las brechas respecto de los países con mayor desempeño en otras regiones. También, se pretenden realizar comparaciones con estudios cienciométricos de la producción científica de América Latina en otras áreas, con el fin de identificar tendencias y valorar de forma ponderada los resultados obtenidos en el presente estudio. 


\section{Agradecimientos}

Los autores agradecen a la Universidad Industrial de Santander y a la Vicerrectoría de Investigación y Extensión de institución, quienes a través del proyecto 1365 brindaron la financiación y los recursos necesarios para que este trabajo se realizara y culminara de manera exitosa; y a la Universidad del Magdalena, por la asesoría de sus profesores.

\section{REFERENCIAS}

[1] WW. Eckerson, Performance Dashboards: Measuring, Monitoring, and Managing Your Business, 2nd ed. USA: Wiley, 2010. ISBN 0470589833.

[2] RM. Bogza, D. Zaharie, "Business intelligence as a competitive differentiator" en IEEE International Conf. on Automation Quality and Testing, Robotics Proc., Cluj-Napoca, 2008, pp. 146-151. DOI: 10.1109 /AQTR.2008.4588724

[3] E. Thomsen, E, "BI's Promised Land," Intell. Enterprise, vol. 6, no. 4, pp. 21-25, 2003.

[4] D. Veset et al., "Worldwide Business Analytics Software 2013-2017 Forecast and 2012 Vendor Shares," IDC, No. 241689, 2013.

[5] RV. Gartner, J. Rivera. (2013, May 20). Gartner Says Business Intelligence/Analytics Is Top Area for CFO Technology Investment Through 2014 [Comunicado de prensa, en línea]. Disponible en: http://goo.gl/rhwrm3

[6] R. Feldman, J. Sanger, The Text Mining Handbook: Advanced Approaches in Analyzing Unstructured Data, 1a ed. NY, USA: Cambridge University Press, 2007. ISBN 9780521836579.

[7] L. Heilig, S. Voß, "A Scientometric Analysis of Cloud Computing Literature," IEEE Trans. on Cloud Comp., vol. 2, no. 3, pp 266-278, Abr. 30, 2014. DOI: 10.1109/TCC.2014.2321168
[8] HH. Tsai, JK. Chiang,TSAI, "E-commerce literature trend forecasting: A study of bibliometric methodology," en 4th International Conference on New Trends in Information Science and Service Science (NISS), Gyeongju, Corea del Sur, 2010, pp. 671 - 676.

[9] AN. Mohamad, MN. Masrek, ARA. Rasam, "A bibliometric analysis on scientific production of Geographical Information System (GIS) in Web of Science," en International Conference of Information and Communication Technology (ICoICT), Bandung, Indonesia, 2013, pp. 264-268. DOI: 10.1109 /ICoICT.2013.6574584

[10] AL. Porter, SW. Cunningham, Tech Mining: Exploiting New Technologies for Competitive Advantage. 1a ed. NJ, USA: Wiley-Interscience, John Wiley \& Sons, Inc., 2005. ISBN-13: 047147567X.

[11] Academic Ranking of World Universities 2014 [Ranking de 500 universidades, en línea]. Disponible en: http://www.shanghairanking.com/ARWU2014.html.

[12] E. Fields. (2013, Dic. 2). Top 10 Trends in Business Intelligence for 2014 [Entrada de blog]. Disponible en: $\mathrm{http} / / /$ goo.gl/eMB9zJ

[13] S. Chaudhuri, U. Dayal, V. Narasayya, "An Overview of Business Intelligence Technology," Comm. Of the ACM, vol. 54, no. 8, pp 88-98, 2011. DOI: $10.1145 / 1978542.1978562$ 